Review Article

\title{
CRIB effector disorder: exquisite function from chaos
}

\author{
Darerca Owen and Helen R. Mott \\ Department of Biochemistry, University of Cambridge, 80 Tennis Court Road, Cambridge \\ CB2 1GA, U.K. \\ Correspondence: Darerca Owen (do202@cam.ac.uk)
}

\begin{abstract}
The CRIB family of small G protein effectors contain significant regions with intrinsic disorder. The $\mathrm{G}$ protein binding regions are contained within these intrinsically disordered regions. Most CRIB proteins also contain stretches of basic residues associated with their $\mathrm{G}$ protein binding regions. The basic region and $G$ protein binding region together allow the CRIB effectors to bind to their cognate $\mathrm{G}$ protein via a dock and coalesce binding mechanism. The basic regions of these proteins take on multiple roles: steering $\mathrm{G}$ protein binding, interacting with elements of the membrane and regulating intramolecular regulatory interactions. The ability of these regions of the CRIBs to undergo multivalent interactions and mediate charge neutralizations equips them with all the properties required to drive liquid-liquid phase separation and therefore to initiate and drive signalosome formation. It is only recently that the structural plasticity in these proteins is being appreciated as the driving force for these vital cellular processes.
\end{abstract}

\section{Abbreviations}

$\mathrm{BR}$, basic region; CRIB, Cdc42/Rac interactive binding; GBD, Gprotein binding domain; GDP, guanosine-5'-diphosphate; GTP, guanosine-5'-triphosphate; IDR, intrinsically disordered region; IDP, intrinsically disordered protein; MoRE, Molecular Recognition Elements; MoRF, Molecular Recognition Feature.

\section{Introduction}

The field of small G protein biology, as much as any other, has been dominated and beautifully informed by the structure-function paradigm. However, more recent re-evaluation has led to the emerging concept that many protein functions lie within intrinsically disordered regions (IDRs) of proteins, especially in signalling proteins (1-3). Signalling proteins are highly modular, often containing multiple, defined, structured domains, which are frequently linked by IDRs. IDRs have the ability to fold into one or more conformations or indeed to remain largely unstructured. This intrinsic disorder 
often results in dynamic binding and allows for multiple partners. IDRs therefore play an important role in assembling subsets of components within signalosomes. These properties have led to the realization that proteins with IDRs are often central hubs of signalling networks and are revealing a new disorder-function model.

\section{Disorder in the CRIB effectors}

A major group of Rho-family small $G$ protein effectors consists of proteins containing a Cdc42/Rac interactive binding motif (CRIB)(4) As their name suggests, these are effector proteins for the Cdc42/Rac subfamily of Rhofamily proteins. The CRIB motif comprises eight conserved residues within a stretch of 16-18 amino acids (I-S-x-P-x(2,4)-F-x-H-x(2)-H-V-G) and forms the consensus region of a larger $G$ protein binding region (GBD). The CRIB motif itself is necessary but not sufficient for high affinity $G$ protein binding and often has alternative roles in regulating the effector proteins themselves.

The CRIB effector proteins fall into several functional protein families but are mainly protein kinases and/or actin regulatory proteins. This reflects the fact that despite the many roles carried out by the Rho-family small $G$ proteins in cells, they are primarily known for their regulation of the actin cytoskeleton (5). Cdc42, Rac1 and RhoA constitute the archetypal trinity of Rho-family G proteins that control the formation of actin driven cellular structures such as filopodia, lamellipodia and stress fibres, crucially often signalling downstream of the master regulator small G protein, Ras. Many CRIB effector family proteins bind to both $\mathrm{Cdc} 42$ and Rac1 but a subset exclusively bind to Cdc42. Cdc42 induces the formation of filopodia by initiating actin remodeling, while Rac1 regulates lamellipodia development, with these structures being key to cell motility (5).

Like the majority of small G proteins, Cdc42 and Rac1 act as molecular switches, which exist in two conformations. They are inactive when bound to GDP but become active when bound to GTP, changing conformation and becoming competent to bind immediate downstream effector proteins, such as the CRIB family. The switch mechanism itself is regulated by a series of proteins which act to turn the switch on or off in response to cellular stimuli. The switch is summarized in Figure 1, which also demonstrates the wealth of structural and biophysical data available for the complexes involved.

Deregulation of these proteins is often associated with the progression of several disease states, including tumour metastasis (6).

The CRIB family of small G protein effectors also falls into the category of proteins containing IDRs. Analysis of various CRIB proteins using disorder prediction software demonstrates significant stretches of disorder in these proteins and these contain the CRIB consensus sequences (Figure 2). In fact it would seem that the common use of the term G protein binding 'domain' is especially inappropriate for the CRIB effectors. Specifically, structural analysis 
of the CRIB containing regions of these proteins, where available, suggests little, if any, native structure when unbound to partner proteins (7-9).

\section{CRIB effector-Cdc42/Rac binding mechanism}

Along with the various informative structures available for the final complexes formed by CRIB effectors and their cognate small G proteins, an increasing amount of information pertaining to their mode of binding has emerged. It is becoming clear that the CRIB proteins adopt a 'dock and coalesce' mechanism, in line with many IDRs, when binding to small GTPases, with many likely using electrostatic steering in the initial docking phase. This mechanism involves coupled binding and folding, whereby the docking segment of the IDR establishes initial contact with its target, often through long range electrostatic interactions, followed by sections of the IDR binding to their target elements within the binding site on the partner protein in the coalescence phase to achieve the final stable complex.

\section{Steering}

For both disordered and ordered proteins, electrostatic attraction between the partners has been observed to drive enhanced binding rate constants (10). This has been well documented for Ras-effector protein complexes, where a two-step association has been observed experimentally and underpins the very high affinity of the complexes (11-14). In a similar manner, the function of a basic region (BR) N-terminal to the CRIB consensus has been extensively studied for (N-)WASP (Figures 3 and 4). Here, the BR has been shown to associate with negatively charged residues on Cdc42, positioning the $\mathrm{N}$ terminal CRIB consensus residues close to their binding site on Cdc42 (Figure 4) and allowing the full CRIB and the remainder of the GBD to zip up with the switch regions of the GTPase, in a mutually stabilizing process ((15-17) and Tetley et al. under review). Our recent study indicated that residues in the BR closest to the CRIB residues have the largest contribution to binding (Tetley et al. under review). In addition, mutational studies on the BR show that a cloud of positive potential in this region is required for steering, rather than individual specific interactions ((15) and Tetley et al. under review). As this region of WASP lies within an IDR, the lack of structure potentially facilitates substitution between the residues, resulting in overall binding only being affected when all of the positive residues are removed. The available structure of WASP bound to Cdc42 ( $p d b$ 1CEE) does not include the residues in the BR that are more distal to the CRIB consensus but their juxtaposition to acidic residues in Cdc42 (including Glu181, which is likewise absent from the structure) means that they could also contribute to electrostatic interactions in the final complex (Figure 5C).

As seen in Figure 3 most other CRIB effector proteins also contain an associated BR, N-terminal to the CRIB motif. Although little experimental analysis of these regions has been undertaken, computational analysis of the 
role of the PAK1 BR suggests that it plays a similar role in electrostatic steering (17).

From Figure 3 it is also apparent that ACK, unlike the other CRIB effectors, does not contain an obvious BR. However our work suggests that ACK also utilizes a dock and coalesce binding mechanism with the initial docking being mediated by the $\mathrm{N}$-terminal region of the ACK GBD in a manner akin to the electrostatic steering or basic region docking demonstrated for Cdc42-WASP and postulated for Cdc42PAK, but differing significantly by being driven by hydrophobic contacts (Figure 5 and (18)). The patch of residues on ACK comprising Leu449, Ser450 and Ala451 contacts Cdc42 via residues in helix $\alpha 5$ and the Cterminus of the G protein (e.g. Glu181). This hydrophobic patch interaction is further extended by Ile $454^{\mathrm{ACK}}$ (see below), which contacts Leu177, lle46, Gly47 and Gly48 ${ }^{\text {Cdc42, }}$ all of which are structurally stable. An intense concentration of hydrophobic potential is found on Cdc42, complementing the $\mathrm{N}$-terminal hydrophobic residues of $\mathrm{ACK}$ and giving it plausible docking potential (18). This hydrophobic potential is also apparent in free Cdc42 in the GTP-bound form and so would be accessible to form an encounter complex (Figure 5).

\section{Coalescence}

Coalescence in IDRs can involve a second search for a new binding subsite, which is limited by the span of the tether between the dock and coalesce segments (19). This often slows the coalescence phase. However, for the CRIB effectors, it would seem likely that the proximity of the BR to the $\mathrm{N}$ terminus of the CRIB allows 'guided' coalescence due to the limited length of the tether, implying that coalescence should occur rapidly (almost immediately) after docking. This is supported by computational analysis (17).

Our recent studies of the thermodynamic contribution of residues of the ACK and WASP GBDs to the complexes formed with Cdc42 indicate several residues that are key to binding and these are centred in both cases on the $\mathrm{CRIB}$ consensus residues. How the individual energetic contributions from these residues map onto the structures of the GBDs is shown in Figure 6. The GBDs are anchored to Cdc42 at each end by hydrophobic interactions. After steering, we envisage the hydrophobic contributions from the residues immediately C-terminal to the BR providing the anchor point for the interface, with the isoleucine of the CRIB consensus being the key residue.

Complex formation between the CRIB effectors and Cdc42 takes an interesting twist at this point. For most IDRs studied, their target binding partner is a structured protein or structured domain. Although small $G$ proteins are highly structured, the switch regions that bind effector proteins are flexible, changing conformation between the active and inactive forms of the proteins. For most small $G$ proteins the switches are relatively mobile in the inactive, GDP-bound 
conformation. In the active conformation, two states exist (state 1 and 2) with one or the other being more populated depending on the $G$ protein. State 2 is the conformation capable of engaging effectors and is the dominant species in Ras when bound to a GTP analogue (20). For Cdc42 (the main target for CRIB effectors), on the contrary, the switches appear to be predominately in state 1 even when bound to a GTP analogue and only become stabilized in the state 2 conformation by the effector itself (21). The mechanism suggested to underpin this involves the effector initially binding loosely, creating a favourable hydrophobic cleft around Phe $37^{\mathrm{Cdc} 42}$ and Val36 ${ }^{\mathrm{Cdc} 42}$. The resulting movement of switch I is then stabilized by Thr35 coordination to both the $\gamma$ phosphate of GTP and to $\mathrm{Mg}^{2+}$. When GDP is bound this cannot occur and the effector complex is transitory; likewise without the effector protein, the active conformation is also transitory (21).

Importantly, the CRIB consensus isoleucine binds to residues on Cdc42 outside the flexible switch regions. This region of Cdc42 is not subject to the rearrangements experienced by the switches and provides a relatively stable platform to initiate the coalesce phase of complex formation and therefore facilitate molecular recognition for the two partners. For example, lle454 ${ }^{\mathrm{ACK}}$ contacts Leu177, Ile46, Gly47 and Gly48 ${ }^{\mathrm{Cdc42}}$ all of which are structurally stable, nucleotide independent residues. Similar contacts are seen between the equivalent CRIB consensus isoleucines in other complexes $(9,22)$. For both ACK and WASP, the first section of the GBD contains a concentration of residues that are energetically vital for binding (Figure 6).

In the model of effector stabilization of state 2, CRIB effector residues in the vicinity of Phe $37^{\mathrm{Cdc} 42}$ and Thr35 ${ }^{\mathrm{Cdc} 42}$ will be of critical importance. These are engaged in the coalescence phase and reside in the more $\mathrm{C}$-terminal sections of the GBDs ((18) and Tetley et al. under review). During the coalesce phase, it seems that the conserved histidines of the CRIB, assisted by other nearby residues, grasp switches I and II and create an environment where Thr35 ${ }^{\mathrm{Cdc} 42}$ can interact with both the $\gamma$ phosphate of GTP and $\mathrm{Mg}^{2+}$ to create a stable complex. This drives further interactions through the CRIB region, completing the major part of coalescence, during which the CRIB residues in ACK and WASP (and presumably other CRIB effectors) position and stabilize the switch loops of Cdc42 in the active, state 2, conformation. The final stages of the coalesce phase would involve hydrophobic interactions with residues Cterminal to the CRIB consensus, which act to stabilize and anchor the Cterminal end of the interface ((18) and Tetley et al. under review).

Thus, unlike many IDR interactions, where the target protein is structured, in the Cdc42-CRIB effector complexes the disordered structure of the effector and the conformational sampling of the target binding interface are together stabilized into the active signal transducing conformation by a reciprocal rigidifying mechanism, likely driven by favourable enthalpic contributions. $A$ somewhat similar situation has previously been reported for Mcl-1-PUMA binding. The small, disordered protein PUMA folds into a helix when bound 
into a groove on the surface of its ordered partner Mcl-1, however Mcl-1 is also observed to undergo conformational rearrangements, closing up around PUMA to stabilize the interaction (23).

\section{Functional Roles of CRIB IDRs in signalling}

\section{WASP Activation in cells}

IDRs play important roles in assembling subsets of components in signalosomes, facilitating construction of reversible cellular assemblies. A good example of the involvement of a CRIB effector in these processes is that of the WASP proteins in actin polymerization. In WASP, the BR and GBD provide a dynamic binding region that is vital in determining the cellular sequelae of WASP interactions. The WASP proteins serve as platforms to integrate multiple signal inputs, with their activity controlled by numerous ligands and through multiple mechanisms with resulting WASP activation stimulating actin polymerization by Arp2/3 (24).

Unstimulated WASP is autoinhibited, with the C-terminal, catalytic VCA region (named for its three sub-regions: verprolin (V) homology, central (C) hydrophobic and acidic (A) regions), bound by part of the GBD region (25). This interaction structures a region spanning residues His249 to Glu295, which includes the end of the CRIB consensus and the $\mathrm{C}$-terminal region of the WASP GBD. The BR and N-terminal WASP GBD residues critical to Cdc42 binding, such as lle233, Ile238, Pro241 and Phe244, are not within this structured region. However, the BR residues are thought to interact with the acidic region (A) of WASP/N-WASP $(26,27)$ due to complementary charge interactions.

To stimulate actin polymerization, the VCA domain must interact with Arp2/3 and so needs to be released from the GBD. The BR initiates this transition by interacting with the negative charge density on Cdc42. This weakens the structure of autoinhibited WASP and opens the N-terminal GBD residues for further interaction with Cdc42. The remaining WASP GBD residues can then exchange intramolecular interactions for contacts with $\mathrm{Cdc} 42$, releasing the VCA domain. Initial docking of Cdc42 and WASP relies on non-selective charge interactions, which could be achieved by WASP residues that are not involved in intramolecular interactions and therefore freely available. Hence, conformational sampling by the GBD, which is likely to be limited in the autoinhibited structure, would be unnecessary in this instance.

$\mathrm{PIP}_{2}$ also plays a role in the activation of the WASP proteins, and N-WASP is synergistically activated by $\mathrm{PIP}_{2}$ and Cdc42 in vitro (28). N-WASP is multivalent for $\mathrm{PIP}_{2}$ binding (26), with multiple $\mathrm{PIP}_{2}$ molecules acting cooperatively in WASP activation, in part because the BR of N-WASP appears again to be a constituent of the autoinhibition complex. Cdc42 significantly changes this co-operativity between N-WASP and $\mathrm{PIP}_{2}$, lowering the activation threshold and allowing lower levels of $\mathrm{PIP}_{2}$ to activate N-WASP. 
Such observations imply that Cdc42 and $\mathrm{PIP}_{2}$ bind to N-WASP simultaneously to relieve VCA autoinhibition, the obvious mechanism being where Cdc42 binds the GBD and some or all of the BR interacts with $\mathrm{PIP}_{2}$.

Naturally this model, where $\mathrm{PIP}_{2}$ binds the $\mathrm{BR}$, would aid membrane localization of N-WASP. PIP ${ }_{2}$ tends to be localized to certain areas of the membrane in nanoclusters (29) and considering that higher local densities of $\mathrm{PIP}_{2}$ activate N-WASP (Papayannopoulos, 2005 \#1291), such sites could provide loci for actin nucleation, even in the absence of Cdc42. Membrane localization would however, also increase the chance of WASP encountering active Cdc42, which is similarly membrane localized, in the cell. The simultaneous binding of $\mathrm{PIP}_{2}$ and active, membrane localized, Cdc42 by WASP appears likely, particularly since the $\mathrm{N}$-terminal region of the BR appears to be less important for Cdc42-WASP binding (Tetley et al. under review) leaving it available for $\mathrm{PIP}_{2}$ interaction. Since the interactions of the $\mathrm{BR}$ are electrostatic they are likely to be quite fluid and, as such, the residues of the BR could switch interactions between the acidic region of WASP, $\mathrm{PIP}_{2}$ and $\mathrm{Cdc} 42$, aided by their intrinsic disorder.

The on- and off-rates of WASP binding to Cdc42 are high ((15) Tetley et al. under review), and individual interactions with partners are likely to be simultaneously transient. However, the concurrent interaction of WASP with $\mathrm{PIP}_{2}$ and active Cdc42, facilitated by its disorder, could stabilize WASP in an open form by reducing the off-rate from the complex. Simultaneous binding at several linked sites is known to produce such an effect (30). The proximity of the BR and the GBD should strengthen the off-rate effect and reduce Cdc42WASP unbinding events. Stable release of the VCA should increase the duration and robustness of Arp2/3 activation.

\section{Other CRIB effectors}

Multiple roles for the disordered BR-CRIB regions are also seen in the PAK proteins. PAK1 also exists in an autoinhibited conformation and possesses a BR (Figure 3). Autoinhibited PAK1 has a structure very similar to that found in WASP, with the GBD and sequences immediately $\mathrm{C}$-terminal folding to give a compact inhibitory switch (IS) domain (31). Unfortunately the BR is missing in these studies, however interaction with Cdc42 is postulated to initiate binding from the N-terminal region of the GBD (residues 70-84) and then proceed through the rest of the GBD, ultimately leading to unfolding of the IS domain and release of the kinase domain. The inclusion of the BR in this scheme would again serve to steer initial recognition using electrostatic interactions between the BR and Cdc42 as for WASP. In the absence of one of its binding partners, PAK1 70-149 is considered to be fully disordered (31), however NMR data for free 75-118 PAK show a helix from 101-108, which is maintained in the complexes formed with both Cdc42 and with the kinase (9, 31). Structure is however obviously limited in the unbound form of the BRCRIB region of PAK1, as it is in WASP. 
A more recent structure of full-length PAK4-Cdc42 also reports an interesting new potential function for the PAK4 BR (Figures 1 and 3). In this structure, which is the first to report structural details for a full-length effector protein, Cdc42 is shown to make extra contacts to PAK4 outside the canonical GBD. Contacts are seen between helix $\alpha 5$ in Cdc42 and the kinase domain of PAK4 and, in addition, the PAK4 BR sits in the substrate binding cleft of PAK4 in the presence of Cdc42, acting as an inhibitor in place of the displaced pseudosubstrate region (32). These data may explain why Cdc42 has not been seen to significantly activate the kinase activity of the type II PAKs (33, 34); it seems rather that Cdc42 acts to prime PAK4 for full activation. If this complex were situated at a membrane, phospholipid interactions could also be present to engage the PAK4 BR and fully activate the complex.

It seems likely that the presence of a BR juxtaposed to the CRIB region in these effectors correlates with the presence of an autoinhibited structure involving the GBD, where the BR can act as a vital motif in breaking up the native tertiary structure in the inactive proteins to ultimately allow GBD coalescence with Cdc42 and activation of the effector.

Very little further data is available regarding the specific regulation of other CRIB effector proteins but all the available information points to the importance of the disorder of the GBD and its ability to interact with multiple partners/ligands.

The MLKs (mixed-lineage kinases) are a subset of CRIB effectors, which belong to the MAP3K family and were the first group of serine/threonine kinases shown to be regulated by an intramolecular SH3-kinase interaction (35). For MLK3 the recognition site for the $\mathrm{SH} 3$ domain is a non-conventional single proline residue that resides lies very close to the BR (Figure 3 ). It is expected that binding of Cdc42 to the CRIB region disrupts the intramolecular SH3-proline interaction, activating the kinase activity.

The CEP (BORG) family of CRIB effectors possess BRs and are also suspected to be subject to autoinhibition, in this case through the Cll region (36). This family of CRIB effectors do not contain any domains of known biochemical activity and are therefore suggested to act structurally, possibly to scaffold interactions involved in regulation of the actin and septin networks.

As noted previously, ACK does not possess a BR (Figure 3) but is also not thought to be autoinhibited in a manner that involves the CRIB region (37), so that the ACK GBD is freely available to associate with Cdc42. The differences between the ACK GBD and the other CRIB effectors and their respective binding mechanisms with Cdc42 likely reflect evolutionary responses to the different roles of the GBDs in the complete effector proteins. 


\section{Potential role for disordered CRIB effectors in the formation of biomolecular condensates}

The unexpected revelation that $\mathrm{P}$ granules in $C$. elegans are actually liquid droplets with all the physical properties of classical liquids (38) and subsequent discoveries that their formation is driven by specific proteins with the capacity to separate from the surroundings by liquid-liquid demixing or phase separation (39), has opened up an entirely new view of cellular organization.

It is now recognized that above a critical concentration certain types of proteins can drive phase separation, resulting in the creation of a protein-rich phase that is in equilibrium with the remaining protein-poor cytoplasm (or nucleoplasm). Even in the very early stages of research into cellular phase transitions it was noted that proteins with intrinsic conformational heterogeneity were in the driving seat (39). The membrane-less compartments, or biomolecular condensates, created by this phase separation act as cellular regions capable of facilitating biochemical reactions due to crowding. These phase separated cellular 'bodies' impact on multiple cellular processes but are often centers for RNA processing, ribosomal biogenesis and stress responses (40). They are also involved in DNA-protein interactions e.g. at centrosomes and mitotic spindles (41). This process also seems particularly active in contributing to the organization of signalling complexes, with phase separated signalosomes existing at the membrane. In fact some of the best studied phase transition systems involve signalling complexes. Signalling from the T-cell receptor, resulting in actin remodeling is a particularly rich source of experimental evidence of phase-phase separation involvement in signal transduction (42).

The Nephrin-Nck-N-WASP signalosome has also been used as a model system to understand the blossoming field of liquid-liquid phase transitions in cellular biology. In this system, interaction between the adhesion receptor Nephrin, the adapter Nck and the CRIB effector N-WASP results in phase separation of the signalling proteins into membrane associated puncta (43). The association of the proteins is mediated by multivalent interactions between the individual components as well as the presence of IDRs in the components, both of which are known to drive phase separation of proteins. Charge neutralization or electrostatic attraction is also a key process in phase separation (44) and the N-WASP basic region is implicated in this process, potentially aiding Nephrin phase separation by virtue of its positive charge (45). The presence of specific modular protein-protein interactions alongside non-specific charge interactions underpin the phase separation of the complex and lead to the formation of membrane puncta presumably representing the signalosome. A similar process has been observed in T-cell receptor signalling also involving N-WASP (42).

As a variety of mechanisms exist to facilitate multivalent polymerization and phase separation, this process likely occurs in many signalling systems. Like 
(N)WASP, many CRIB effectors contain both IDRs and charged regions, making them potential candidates to take part in or even drive these processes (Figures 2 and 3). The small G protein direct regulators of the CRIB effectors are also likely participants in this procedure. Lipid anchored Ras has been shown to organize into dynamic clusters at the membrane, containing 6-8 Ras molecules (46). The formation of K-Ras nanoclusters has been elegantly demonstrated to ensure MAPK output from EGF stimulation is binary (47). Similarly Rac1, which binds a subset of CRIB effector proteins, has also been shown to form nanoclusters, which interestingly are larger ( 200nm) and contain 50 Rac1 molecules. The clusters are heterogeneous in terms of protein composition and include downstream effector proteins, which are then important for immobilizing Rac1 into the clusters (48). The recent observation of nanoclustered Cdc42, at least in yeast, suggests that this will be a general feature for small GTPases (49). The small GTPase nanoclusters may well constitute the marker for effector protein recruitment (and potentially further associated proteins) to the membrane, which, if the correct type of proteins are involved e.g. CRIB effectors, will then facilitate phase separation at the membrane and the formation of signalosomes.

It appears, at least in the T-cell receptor system, that phosphatases may be excluded from the phase separated cluster while kinases (which are abundant in the CRIB family effectors) are enriched. Furthermore, phosphorylation by the kinases would be expected to enhance phase separation and therefore increase output from the signalosome in a positive feed-forward manner (42).

\section{Therapeutic possibilities: AttACKing IDRs}

$80 \%$ of proteins involved in processes driving cancers contain IDRs (50) and $60 \%$ of proteins associated with cardiovascular and neurodegenerative disorders can also be classified as IDPs (51), so there is an increasing interest in IDRs as therapeutic targets.

Most studies concentrate on screening for small molecules that bind the IDP and change its activity, for example by stabilizing their native disorder or inhibiting their interaction with partners. This approach has had some success, with several inhibitors of c-Myc/Max interaction identified that disrupt the c-Myc/Max interaction by binding to monomeric c-Myc and stabilizing conformations incompatible with Max heterodimerization (52).

An alternative strategy could involve using peptides based on the IDR itself to inhibit its interaction with partners. Peptide based therapeutic strategies have long been the poor relation of small molecule therapeutics, especially for intracellular targets. However, advances in stabilization and delivery have brought new hopes for this modality (53).

IDPs mediate protein-protein interactions through a variety of elements. Short linear motifs (SLiMs) are generally between 3-10 amino acids and usually 
exist in extended conformations in complex with their partners (54). Molecular recognition Elements (MoREs) or Molecular recognition features (MoRFs) are slightly longer sequences of 10-70 amino acids. These often undergo disorder to order transitions on binding and adopt thermodynamically stable, welldefined structures in the context of the complex. When bound to their partner, MoRFs may form alpha-helices (a-MoRFs), beta-strands ( $\beta-M o R F s)$, irregular structure (i-MoRFs) or combinations of these elements (complex-MoRFs) (55). SLiMS and MoREs/MoRFs are therefore molecular recognition elements that capture the appropriate binding partner with high specificity. Therefore, as IDRs utilize various peptide motifs to mediate protein-protein interactions, they are a rich potential source of parent sequences for peptide therapeutics.

One disadvantage of using the IDR itself as a starting point for a peptide therapeutic is that although IDPs bind their target with high specificity they tend to employ multivalent binding with each element having relatively low affinity for its partner. In fact the CRIB proteins, when binding to their cognate small $\mathrm{G}$ protein, go against this conventional paradigm, displaying high specificity with high affinity (low $\mathrm{nM}$ ) mediated by a single site. With the luxury of both qualities, these CRIB effector IDRs are undoubtedly attractive as progenitor peptides.

Our recent studies have dissected the kinetics and thermodynamics of the binding of both WASP and ACK GBDs to Cdc42 ((18) and Tetley et al. under review). WASP is the tighter binder of the two but removal of the WASP BR leaves ACK with the higher relative affinity. The corollary of this suggests that the ACK GBD with a BR would be expected to bind even more tightly, likely with picomolar affinity. Although this sequence may be too long to act as a therapeutic itself, it would provide an excellent starting point for further maturation.

\section{Conclusions}

A model of Cdc42-CRIB effector binding involving a dock and coalesce mechanism initiated by electrostatic steering is evolving, where the interaction with the effector protein stabilizes switch I in the active conformation bringing the effector GBD and the flexible parts of Cdc42 into a stable quaternary structure. Energetically important residues in the CRIB effectors are primarily concentrated in the $\mathrm{N}$-terminus and CRIB consensus of the GBD. WASP and ACK bind initially to Cdc42 via this $\mathrm{N}$-terminal energetic hotspot where the complementary Cdc42 structure is more rigid regardless of activation state and therefore provides a stable sub-site to initiate coalescence. In the case of WASP, PAK1 and likely the majority of CRIB effectors, this is assisted by a poly-lysine directed electrostatic steering mechanism, with the steering section playing little part in the final complex. In ACK, encounter would be directed by hydrophobic interactions that also remain as part of the final complex. 
The electrostatic steering regions and GBDs of the CRIB effectors are also often involved in intramolecular regulation and take part in multiple interactions with a variety of partner proteins. The intrinsic disorder observed in the regions of the CRIB effectors that contain these binding elements equips them with important biophysical characteristics, the biological consequences of which are only just being revealed. The CRIB effectors are likely initiators of signalosome formation via multiple modular interactions with other components and play a key role in phase separation underpinned by charge interactions leading to final signalosome assembly.

\section{Acknowledgements}

The authors would like to thank Dr Katherine Stott and Dr George Tetley for helpful discussions.

\section{Declaration of Interests}

The authors declare that they have no competing interests associated with this manuscript.

\section{Funding Information}

Work discussed in this manuscript was supported by an MRC CASE Studentship (MR/K017101/1) to D.O. and H.R.M.

\section{Author Contribution}

D.O. and H.R.M. wrote the paper and made all figures.

\section{References}

1. lakoucheva LM, Brown CJ, Lawson JD, Obradović Z, Dunker AK. Intrinsic Disorder in Cell-signaling and Cancer-associated Proteins. J Mol Biol. 2002;323(3):573-84.

2. Wright $P E$, Dyson HJ. Intrinsically disordered proteins in cellular signalling and regulation. Nat Rev Mol Cell Biol. 2015;16(1):18-29.

3. Wu H, Fuxreiter M. The Structure and Dynamics of Higher-Order Assemblies: Amyloids, Signalosomes, and Granules. Cell. 2016;165(5):105566.

4. Burbelo PD, Drechsel D, Hall A. A Conserved Binding Motif Defines Numerous Candidate Target Proteins For Both Cdc42 and Rac GTPases. J Biol Chem. 1995;270(49):29071-4.

5. Ridley AJ. Rho GTPase signalling in cell migration. Curr Opin Cell Biol. 2015;36:103-12.

6. Arias-Romero LE, Chernoff J. Targeting Cdc42 in cancer. Expert Opin Ther Targets. 2013;17(11):1263-73.

7. Rudolph MG, Bayer P, Abo A, Kuhlmann J, Vetter IR, Wittinghofer A. The Cdc42/Rac interactive binding region motif of the Wiskott Aldrich syndrome 
protein (WASP) is necessary but not sufficient for tight binding to Cdc42 and structure formation. J Biol Chem. 1998;273(29):18067-76.

8. Mott HR, Owen D, Nietlispach D, Lowe PN, Manser E, Lim L, et al. Structure of the small $G$ protein Cdc42 bound to the GTPase-binding domain of ACK. Nature. 1999;399(6734):384-8.

9. Morreale A, Venkatesan M, Mott HR, Owen D, Nietlispach D, Lowe PN, et al. Structure of Cdc42 bound to the GTPase binding domain of PAK. Nat Struct Biol. 2000;7(5):384-8.

10. Zhou HX, Pang X. Electrostatic Interactions in Protein Structure, Folding, Binding, and Condensation. Chem Rev. 2018;118(4):1691-741.

11. Sydor JR, Engelhard M, Wittinghofer A, Goody RS, Herrmann C.

Transient kinetic studies on the interaction of ras and the Ras-binding domain of c-Raf-1 reveal rapid equilibration of the complex. Biochemistry.

1998;37(40):14292-9.

12. Linnemann T, Geyer M, Jaitner BK, Block C, Kalbitzer HR, Wittinghofer A, et al. Thermodynamic and kinetic characterization of the interaction between the Ras binding domain of AF6 and members of the Ras subfamily. J Biol Chem. 1999;274(19):13556-62.

13. Spoerner M, Herrmann C, Vetter IR, Kalbitzer HR, Wittinghofer A. Dynamic properties of the Ras switch I region and its importance for binding to effectors. Proc Natl Acad Sci U S A. 2001;98(9):4944-9.

14. Linnemann T, Kiel C, Herter P, Herrmann C. The activation of RalGDS can be achieved independently of its Ras binding domain. Implications of an activation mechanism in Ras effector specificity and signal distribution. J Biol Chem. 2002;277(10):7831-7.

15. Hemsath L, Dvorsky R, Fiegen D, Carlier MF, Ahmadian MR. An electrostatic steering mechanism of Cdc42 recognition by Wiskott-Aldrich syndrome proteins. Mol Cell. 2005;20(2):313-24.

16. Zhou H-X, Pang X, Lu C. Rate constants and mechanisms of intrinsically disordered proteins binding to structured targets. Phys Chem Chem Phys. 2012;14(30):10466-76.

17. Pang X, Zhou H-X. Mechanism and rate constants of the Cdc42 GTPase binding with intrinsically disordered effectors. Proteins-Structure Function and Bioinformatics. 2016;84(5):674-85.

18. Tetley GJN, Mott HR, Cooley RN, Owen D. A dock and coalesce mechanism driven by hydrophobic interactions governs $\mathrm{Cdc} 42$ binding with its effector protein ACK. J Biol Chem. 2017;292(27):11361-73.

19. Ubbink $M$. The courtship of proteins: understanding the encounter complex. FEBS Lett. 2009;583(7):1060-6.

20. Geyer M, Schweins T, Herrmann C, Prisner T, Wittinghofer A, Kalbitzer HR. Conformational transitions in p21(ras) and in its complexes with the effector protein Raf-RBD and the GTPase activating protein GAP.

Biochemistry. 1996;35(32):10308-20.

21. Phillips MJ, Calero G, Chan B, Ramachandran S, Cerione RA. Effector proteins exert an important influence on the signaling-active state of the small GTPase Cdc42. J Biol Chem. 2008;283(20):14153-64.

22. Abdul-Manan N, Aghazadeh B, Liu GA, Majumdar A, Ouerfelli O, Siminovitch KA, et al. Structure of Cdc42 in complex with the GTPase-binding 
domain of the 'Wiskott-Aldrich syndrome' protein. Nature.

1999;399(6734):379-83.

23. Rogers JM, Oleinikovas V, Shammas SL, Wong CT, De Sancho D, Baker $\mathrm{CM}$, et al. Interplay between partner and ligand facilitates the folding and binding of an intrinsically disordered protein. Proc Natl Acad Sci U S A. 2014;111(43):15420-5.

24. Millard TH, Sharp SJ, Machesky LM. Signalling to actin assembly via the WASP (Wiskott-Aldrich syndrome protein)-family proteins and the Arp2/3 complex. Biochem J. 2004;380:1-17.

25. Kim AS, Kakalis LT, Abdul-Manan M, Liu GA, Rosen MK. Autoinhibition and activation mechanisms of the Wiskott-Aldrich syndrome protein. Nature. 2000;404(6774):151-8.

26. Papayannopoulos V, Co C, Prehoda KE, Snapper S, Taunton J, Lim WA. A polybasic motif allows N-WASP to act as a sensor of PIP2 density. Mol Cell. 2005;17(2):181-91.

27. Suetsugu S, Miki H, Takenawa T. Identification of another actin-related protein (Arp) 2/3 complex binding site in neural Wiskott-Aldrich syndrome protein (N-WASP) that complements actin polymerization induced by the Arp2/3 complex activating (VCA) domain of N-WASP. J Biol Chem. $2001 ; 276(35): 33175-80$.

28. Rohatgi R, Ma L, Miki H, Lopez M, Kirchhausen T, Takenawa T, et al. The interaction between N-WASP and the Arp2/3 complex links Cdc42-dependent signals to actin assembly. Cell. 1999;97(2):221-31.

29. Wang J, Richards DA. Segregation of PIP2 and PIP3 into distinct nanoscale regions within the plasma membrane. Biology Open.

2012;1(9):857-62.

30. Flock T, Weatheritt RJ, Latysheva NS, Babu MM. Controlling entropy to tune the functions of intrinsically disordered regions. Curr Opin Struct Biol. 2014;26:62-72.

31. Lei M, Lu WG, Meng WY, Parrini MC, Eck MJ, Mayer BJ, et al. Structure of PAK1 in an autoinhibited conformation reveals a multistage activation switch. Cell. 2000;102(3):387-97.

32. Ha BH, Boggon TJ. CDC42 binds PAK4 via an extended GTPase-effector interface. Proc Natl Acad Sci U S A. 2018;115(3):531-6.

33. Abo A, Qu J, Cammarano MS, Dan CT, Fritsch A, Baud V, et al. PAK4, a novel effector for $\mathrm{Cdc} 42 \mathrm{Hs}$, is implicated in the reorganization of the actin cytoskeleton and in the formation of filopodia. EMBO J. 1998;17(22):6527-40. 34. Baskaran Y, Ng YW, Selamat W, Ling FTP, Manser E. Group I and II mammalian PAKs have different modes of activation by Cdc42. EMBO Reports. 2012;13(7):653-9.

35. Zhang $\mathrm{H}$, Gallo KA. Autoinhibition of mixed lineage kinase 3 through its Src homology 3 domain. J Biol Chem. 2001;276(49):45598-603.

36. Hirsch DS, Pirone DM, Burbelo PD. A new family of Cdc42 effector proteins, CEPs, function in fibroblast and epithelial cell shape changes. J Biol Chem. 2001;276(2):875-83.

37. Lin Q, Wang J, Childress C, Yang WN. The activation mechanism of ACK1 (activated Cdc42-associated tyrosine kinase 1). Biochem J.

2012;445:255-64. 
38. Brangwynne CP, Eckmann CR, Courson DS, Rybarska A, Hoege C, Gharakhani J, et al. Germline P Granules Are Liquid Droplets That Localize by Controlled Dissolution/Condensation. Science. 2009;324(5935):1729-32. 39. Brangwynne CP, Tompa P, Pappu RV. Polymer physics of intracellular phase transitions. Nature Physics. 2015;11(11):899-904.

40. Mitrea DM, Kriwacki RW. Phase separation in biology; functional organization of a higher order. Cell Commun Signal. 2016;14:1.

41. Jiang $\mathrm{H}$, Wang S, Huang $\mathrm{Y}, \mathrm{He} X, \mathrm{Cui} H$, Zhu $X$, et al. Phase transition of spindle-associated protein regulate spindle apparatus assembly. Cell. 2015;163(1):108-22.

42. Su XL, Ditlev JA, Hui EF, Xing WM, Banjade S, Okrut J, et al. Phase separation of signaling molecules promotes $T$ cell receptor signal transduction. Science. 2016;352(6285):595-9.

43. Banani SF, Lee HO, Hyman AA, Rosen MK. Biomolecular condensates: organizers of cellular biochemistry. Nat Rev Mol Cell Biol. 2017;18(5):285-98. 44. Bergeron-Sandoval LP, Safaee N, Michnick SW. Mechanisms and Consequences of Macromolecular Phase Separation. Cell. 2016;165(5):106779.

45. Pak CW, Kosno M, Holehouse AS, Padrick SB, Mittal A, Ali R, et al. Sequence Determinants of Intracellular Phase Separation by Complex Coacervation of a Disordered Protein. Mol Cell. 2016;63(1):72-85.

46. Plowman SJ, Muncke C, Parton RG, Hancock JF. H-ras, K-ras, and inner plasma membrane raft proteins operate in nanoclusters with differential dependence on the actin cytoskeleton. Proc Natl Acad Sci U S A. 2005;102(43):15500-5.

47. Tian TH, Harding A, Inder K, Plowman S, Parton RG, Hancock JF. Plasma membrane nanoswitches generate high-fidelity Ras signal transduction. Nat Cell Biol. 2007;9(8):905-U60.

48. Remorino A, De Beco S, Cayrac F, Di Federico F, Cornilleau G, Gautreau A, et al. Gradients of Rac1 Nanoclusters Support Spatial Patterns of Rac1 Signaling. Cell Rep. 2017;21(7):1922-35.

49. Sartorel E, Ünlü C, Jose M, Massoni-Laporte A, Meca J, Sibarita J-B, et al. Phosphatidylserine and GTPase activation control Cdc42 nanoclustering to counter dissipative diffusion. Mol Biol Cell. 2018;29(11).

50. lakoucheva LM, Brown CJ, Lawson JD, Obradovic Z, Dunker AK. Intrinsic disorder in cell-signaling and cancer-associated proteins. J Mol Biol. 2002;323(3):573-84.

51. Uversky VN, Oldfield CJ, Dunker AK. Intrinsically disordered proteins in human diseases: introducing the D2 concept. Annu Rev Biophys. 2008;37:215-46.

52. Joshi P, Vendruscolo M. Druggability of Intrinsically Disordered Proteins. Adv Exp Med Biol. 2015;870:383-400.

53. Mott HR, Owen D. Bioblockades join the assault on small G protein signalling. Semin Cancer Biol. 2018.

54. Van Roey K, Uyar B, Weatheritt RJ, Dinkel H, Seiler M, Budd A, et al. Short Linear Motifs: Ubiquitous and Functionally Diverse Protein Interaction Modules Directing Cell Regulation. Chem Rev. 2014;114(13):6733-78. 
55. van der Lee R, Buljan M, Lang B, Weatheritt RJ, Daughdrill GW, Dunker $\mathrm{AK}$, et al. Classification of intrinsically disordered regions and proteins. Chem Rev. 2014;114(13):6589-631.

56. Owen D, Mott HR, Laue ED, Lowe PN. Residues in Cdc42 that specify binding to individual CRIB effector proteins. Biochemistry. 2000;39(6):124350.

57. Kozlowski LP, Bujnicki JM. MetaDisorder: a meta-server for the prediction of intrinsic disorder in proteins. BMC Bioinformatics. 2012;13.

\section{Figure Legends}

Figure 1: The GTPase cycle of Cdc42: Cartoon representations are shown of Cdc42 in the GDP and GTP bound forms (pdb entries 1A4R and 2QR2 respectively). Cdc42 is shown as a cartoon in cyan and the nucleotide in a stick representation in red. Examples structures of Cdc42 in complex with its regulator are shown: Cdc42-Dbs (pdb entry $1 \mathrm{KZ7}$ ), the $\mathrm{DH}$ and $\mathrm{PH}$ domains from Dbs are shown in purple; Cdc42p50 RhoGAP (pdb entry 1AM4), the GAP domain of p50 RhoGAP is shown in green); Cdc42-RhoGDI1 (pdb entry 1DOA), RhoGDI1 is shown in pink and the geranyl geranyl group attached to Cdc42 is shown as a space fill in dark pink. Cdc42-CRIB effectors complexes are shown wherever structures are available: Cdc42-WASP (pdb $1 \mathrm{CEE}$ ), the WASP GBD is in dark orange; Cdc42-ACK GBD (pdb $1 \mathrm{CF} 4), A C K$ is shown in yellow; Cdc42-PAK1 (pdb 1E0A), the PAK1 GBD is shown in pale orange; Cdc42-PAK6 (pdb 2ODB), the PAK6 GBD is shown in pale orange; Cdc42-PAK4 (pdb 5UPK), PAK4 is shown in pale orange. All other CRIB proteins in the family are listed for completion. The $K_{\mathrm{d}}$ of the Cdc42-WASP interaction is $1 \mathrm{nM}, \mathrm{Cdc} 42-$ PAK1 is 20nM and Cdc42-ACK is $30 \mathrm{nM}$ as measured by scintillation proximity assays (56). The $k_{\text {on }}$ and $k_{\text {off }}$ rates for Cdc42-WASP are $0.793 \mu \mathrm{M}^{-1} \mathrm{~s}^{-1}$ and $19.9 \mathrm{~ms}^{-1}$ respectively. The $k_{\text {on }}$ and $k_{\text {off }}$ rates for Cdc42-ACK are $0.224 \mu \mathrm{M}^{-1} \mathrm{~s}^{-1}$ and $27.8 \mathrm{~ms}^{-1}$ respectively as measured by biolayer interferometry (18).

\section{Figure 2: Disorder prediction plots for selected CRIB effector} proteins: Protein sequences were submitted to the Genesilico MetaDisorder Service for analysis (57). Predictions for both ACK and MRCK $\alpha$ were performed in two halves due to the residue limit of the predictions. For clarity 4 plots, corresponding to predictions from the final metapredictions are shown: MetaDisorder (blue), MetaDisorder3D (black), MetaDisorderMD (green) and MetaDisorderMD2 (orange). Protein sequences submitted for analysis were as follows: ACK (Q07912), WASP (P42768), N-WASP (O00401), PAK1 (Q13153), CEP1 (Q00587), SPEC1 (Q9NRR8), MLK2 (Q02779) and MRCK $\alpha$ 
(Q5VT25). The position of the first conserved lle of the CRIB consensus sequence for each protein is indicated by a red asterisk.

Figure 3: Alignment of the CRIB consenus residues of CRIB effector proteins and their basic regions. Uniprot accession numbers for all proteins are shown, as are the residue ranges. Basic residues preceding the CRIB consensus are highlighted in red. The $\mathrm{SH} 3$ recognition proline in MLK3 is highlighted in green.

Figure 4: Model of the dock and coalesce binding mechanism of Cdc42WASP. The model shows the van der Waals surface of Cdc42 in the Cdc42WASP structure (pdb entry 1CEE) as a solid in grey, with acidic residues (Asp and Glu) coloured pink. Residues Glu49 and Glu178 (which are involved in electrostatic WASP docking) are coloured red. The WASP GBD is shown as a cartoon coloured green, with the BR coloured blue. The final panel is rotated $\sim 45^{\circ}$ to display more of the elements of the final complex.

Figure 5: Docking regions of Cdc42-ACK and Cdc42-WASP. (A) Van der Waals surface of the Cdc42-ACK structure (pdb entry 1CF4). The van der Waals surface of Cdc42 is solid and shown in grey, with hydrophobic residues (Ala, Gly, Val, Ile, Leu, Phe and Met) coloured orange. ACK residues 448-489 are shown as a blue mesh. (B) As in A, but with ACK removed to show the hydrophobic potential of Cdc42 in the ACK binding site. (C) Van der Waals surface of Cdc42-WASP structure (pdb entry 1CEE). The van der Waals surface of Cdc42 is again solid and shown in grey, with acidic residues (Asp and Glu) coloured pink. Residues Glu49 and Glu178 (which are involved in electrostatic WASP docking) are coloured red. Potential electrostatic interactions from residues not contained in the structure are indicated as cartoon representations where positively charged residues are coloured blue and negatively charged residues are red. The relevant residues are also labelled. (D) As in C, but with WASP removed to show the negatively charged residues of Cdc42 around the WASP docking site.

Figure 6: Energetic contributions of ACK and WASP GBD residues to Cdc42 complex formation. (A) The van der Waals surface of WASP residues $225-275$ as a grey mesh with the protein backbone in black. The structure of WASP shown is the conformation it adopts when in complex with Cdc42 ((22) and pdb entry 1CEE). Residues involved in interaction with Cdc42 are coloured with respect to the fold-change in $K_{d}$ upon mutation to alanine as follows: green for 5-15-fold increase, yellow for 15-40-fold increase, orange for 40-100-fold increase, red for a $>100$-fold increase. The amino acid sequence for this region is shown below the structural representation, with residues coloured in the same manner and with font size also indicating relative energetic contribution to complex formation. Accession number for WASP: P42768. (B) The van der Waals surface of ACK residues 448-489 as a grey mesh with the protein backbone in black. The structure of ACK shown is the conformation it adopts when in complex with Cdc42 ((8) and pdb entry 1CF4). Residues involved in interaction with Cdc42 are coloured as in A. The 
amino acid sequence for this region is shown below the structural representation, with residues coloured as in A. Accession number for ACK: Q07912. 
Figure 1

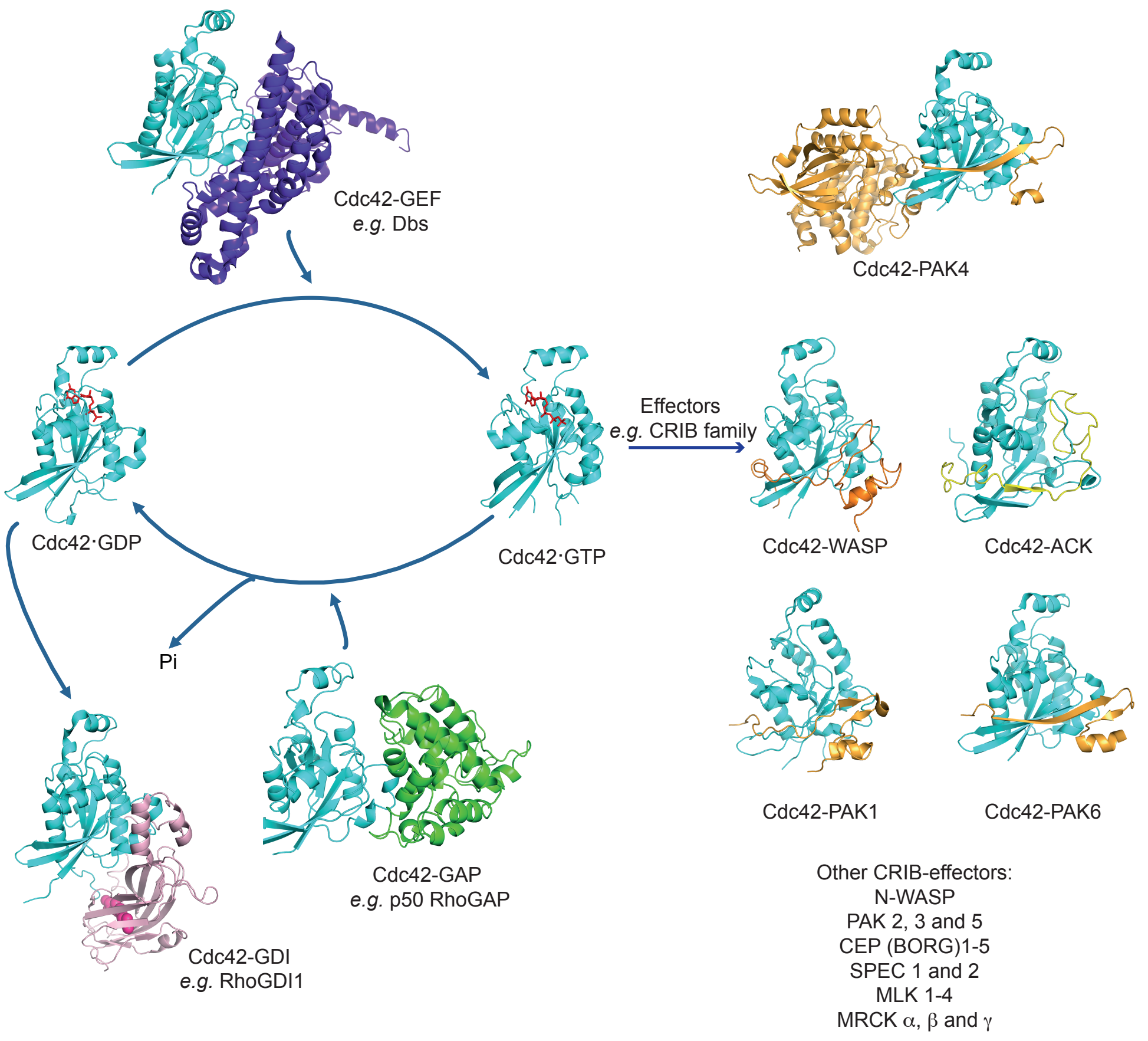


Figure 2

ACK-N

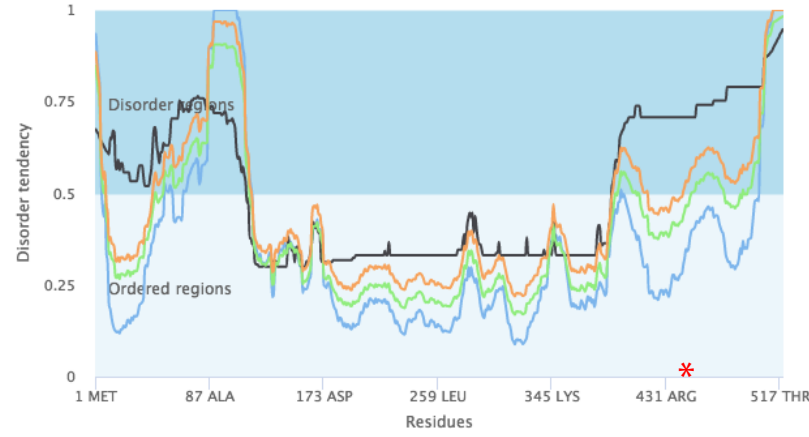

WASP

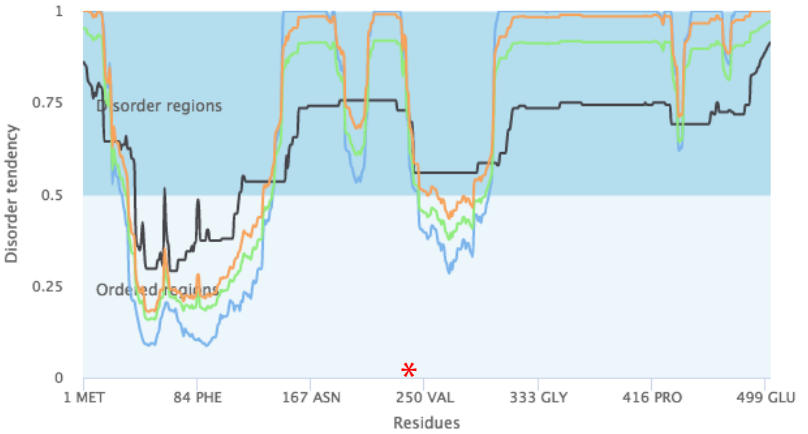

\section{PAK1}

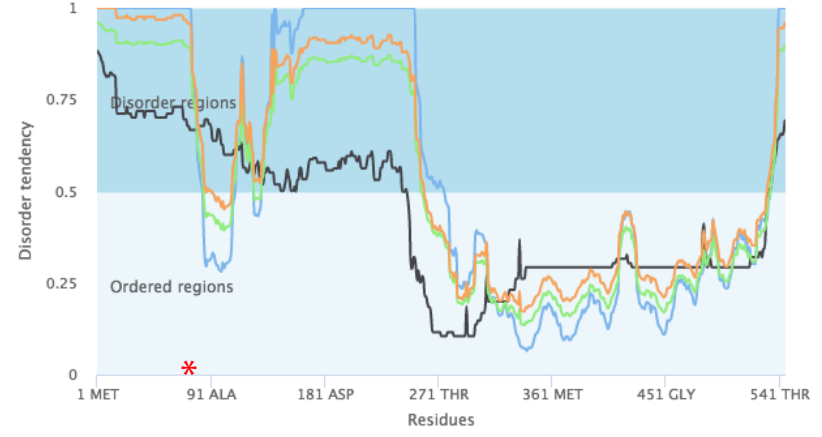

\section{SPEC1}

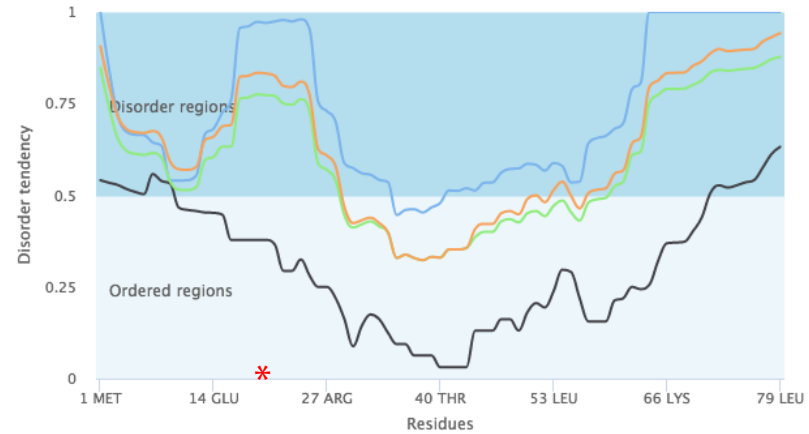

MRCK $\alpha-\mathrm{N}$

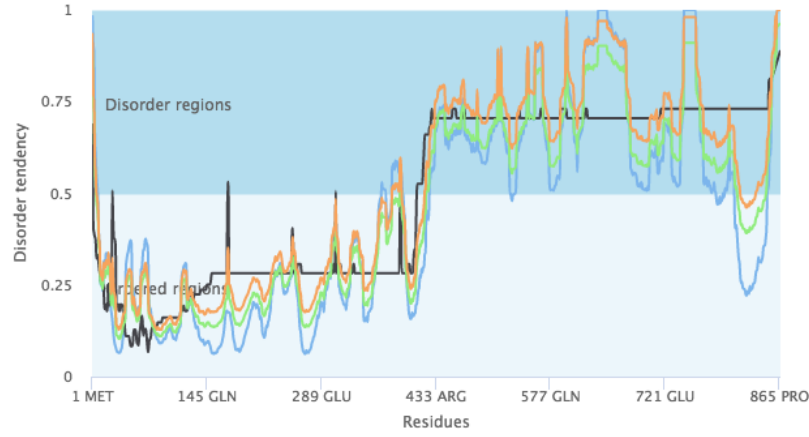

ACK-C

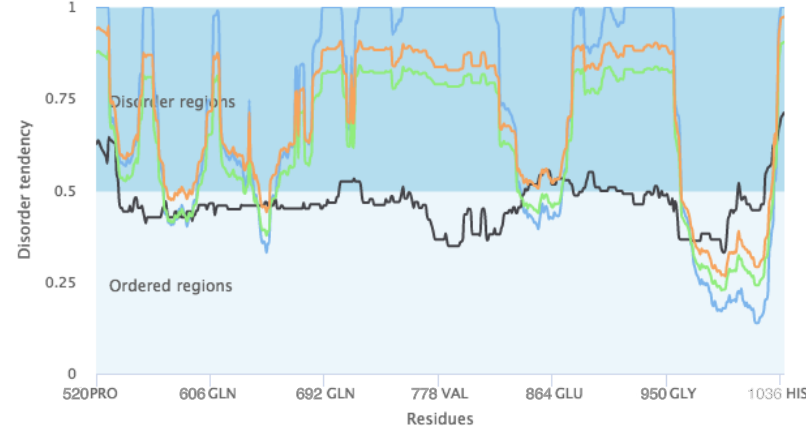

N-WASP

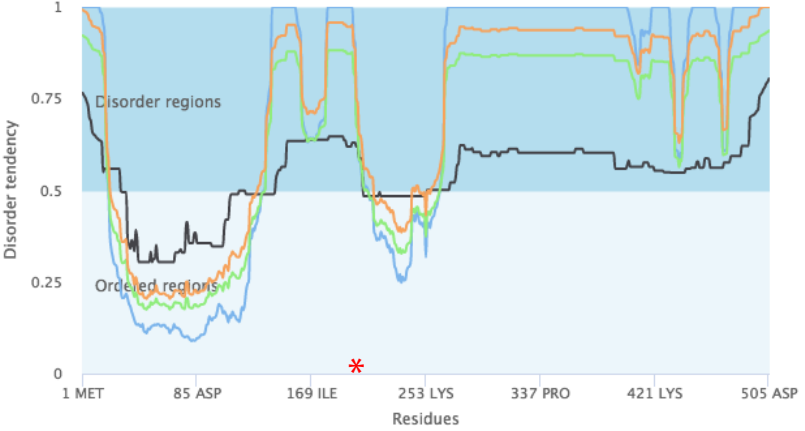

CEP1

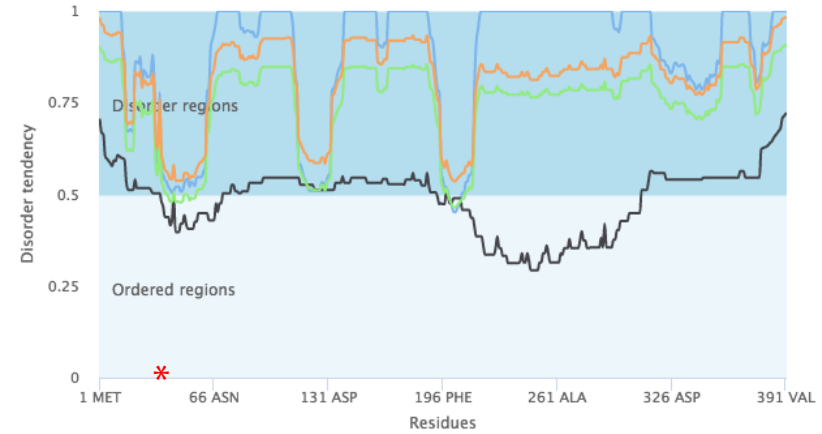

MLK2

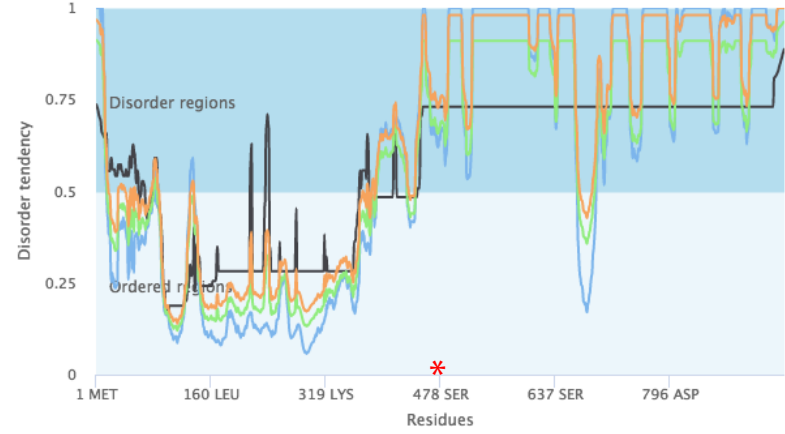

MRCK $\alpha-C$

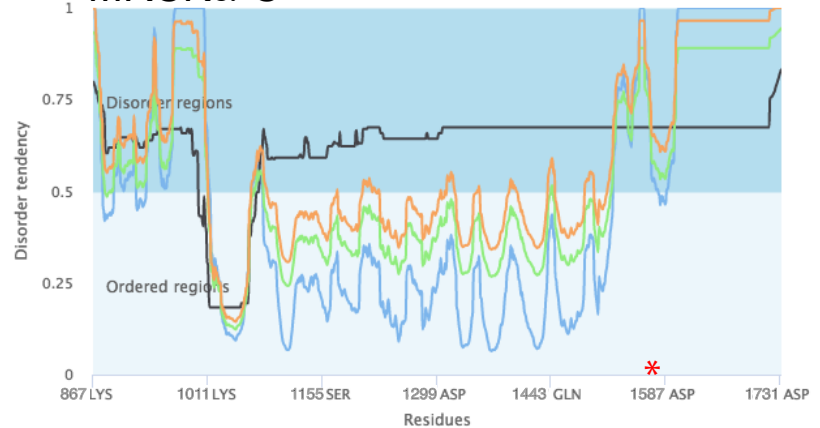


Figure 3

ACK (007912:419-489) WASP ( P $42768: 225-275$ ) N-WASP (000401:186-245) PAK1 (Q13153:63-120) PAK2 (Q13177:62-120) PAK3 (075914:58-129) PAK 4 (096013:4-50) PAK5 (Q9P286:4-50) PAK6 (Q9NQU5: 4-50) CEP 1 ( Q00587: 30-90) CEP2 (014613: 9-68) CEP 3 (Q9UKI 2 : 15-84) CEP 4 (Q9H3Q1: 15-83) CEP5 (Q6NZY 7: 13-80) SPEC 1 (Q9NRR 8 : 5-79) SPEC2 (Q9NRR3 : 15-84) MLK1 (P80192:499-560) MLK1 (P80192: 499-560) MLK2 (002779:448-517) (1) MLK4 (Q5TCX8: 494-560) MRCKa (Q5VT25: 1541-1609) MRCK $\beta$ (Q9Y5S2 : 1553-1621) MRCKY (Q6DT 37: 1407-1475)

CRIB consensus

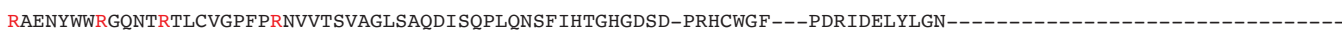

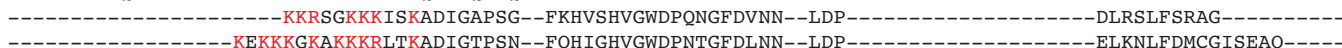

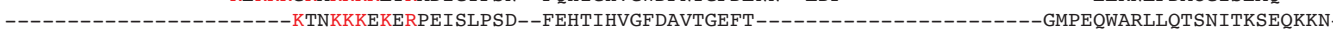
-----------------------KGSKKKEKERPEISPPSD--FEHTI IHVGFDAVTGEFT------------------------GMPEQWARLLQTSNITKLEQKKNP ----------------------KTNKKKEKERPEISLPSD--FEHT IHVGFDAVTGEFTPD--LYG-------SQMCPGKLPEGIPEQWARLLQTSKLEQKK-----

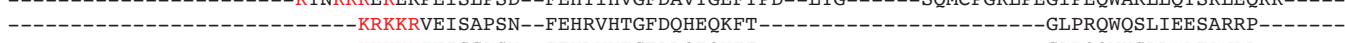

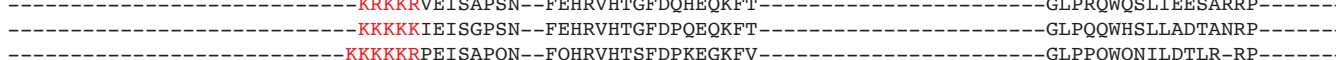

-------------------------KRRLTADMISHPLGD-FRHTMHVGRGG--DVFGDTSFLSNHGGSSGSTH---------RSPRSFLAKK-LQLVR----------------KRGSRKGKKEKLRDLLSSDMISPPLGD-FRHTIHIGSGGGSDMFGDISFLQGKFHLLPGTMVEGPEEDGT-

------------_KGKKKLRDILSPDMISPPLGD-FRHTIHIGKEGQHDVFGDISFLQGNYELLPGNOEKAHLGQFPGHNEF--KRRSRADLTAEMISAPLGD-FRHTMHVGRAG--DAFGDTSFLNSKAGEPDGESLDEOPSSSSSKRSLLSRK-F--

------------_-_---KKRPDRGALSISAPLGD-FRHTLHVGRGG--DAFGDTSFLSRHGGGPPPEPRAPPAGAPRSPPPPAVPQ-SA-------------------KPOPKKKRRRIDRTMIGEPMN--FVHLTH IGSGE--MGAGDGLAMTGAVEQM-------RSKGNRDRPWSNSRG-L-----------------------KRRRRIDRSMIGEPTN--FVHTAHVGSGD--LFSGMNSV-SS IONOM--------QSKGGYGGGMPANVQ-MOLVDTKAG------- KPRVRKRKGNERR -------PHVRRRRGTFKRSKLRARDGGERISMPLD--FKHRITVQASPGLDRRRNV--FEV------G---PG-----DSPTFPRFRA-IQLEPAEP-----------KVKKRKGKFKRSRLKLKDGHRISLPSD--FQHKITVQASPNLDKRRSL--NSS------SSSPPS-----SPTMMPRLRA-IQLT----------KRRYSFRVPEEERMQQRREMLRDPEMRNKLISNPTN--FNHIAHMGPGDGIQILK-D--LPM-------NPRPQESRTVFS-

-----KRRFVFKVPEEERLQQRREMLRDPELRSKMISNPTN--FNHVAHMGPGDGMQVLM-D--LPL------SAVPPSQEERPG------------------------KRRFFFRVSEEQOKOQRREMLKDPFVRSKLISPPTN--FNHLVHVGPANGRPGAR -D--KSP-------APEEKGRVARGS-

ISxPxxxxFxHXxHVG 
Figure 4

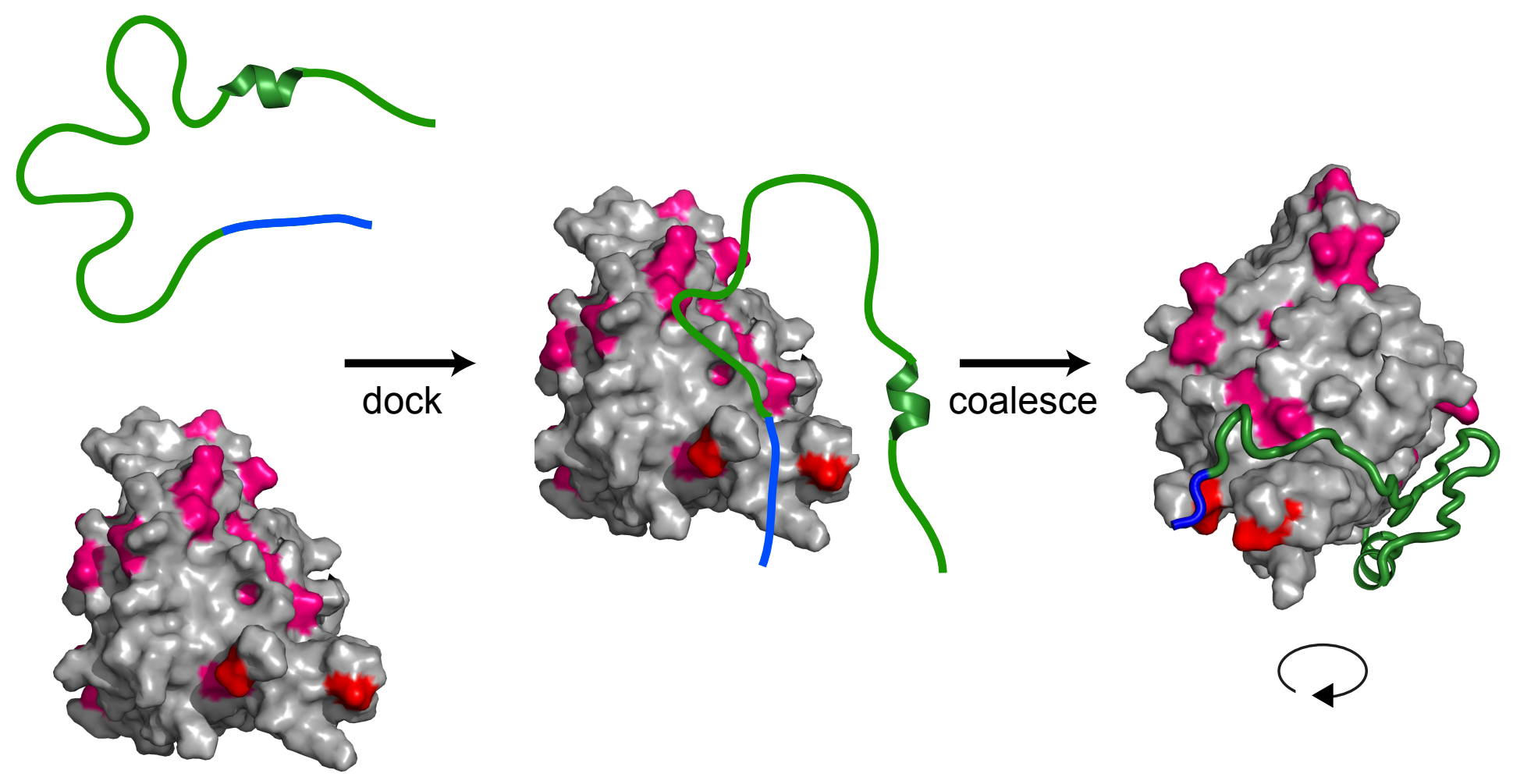


Figure 5

A

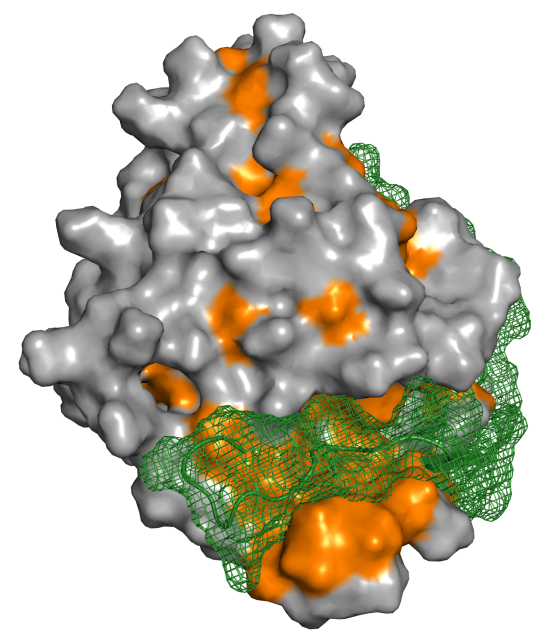

C

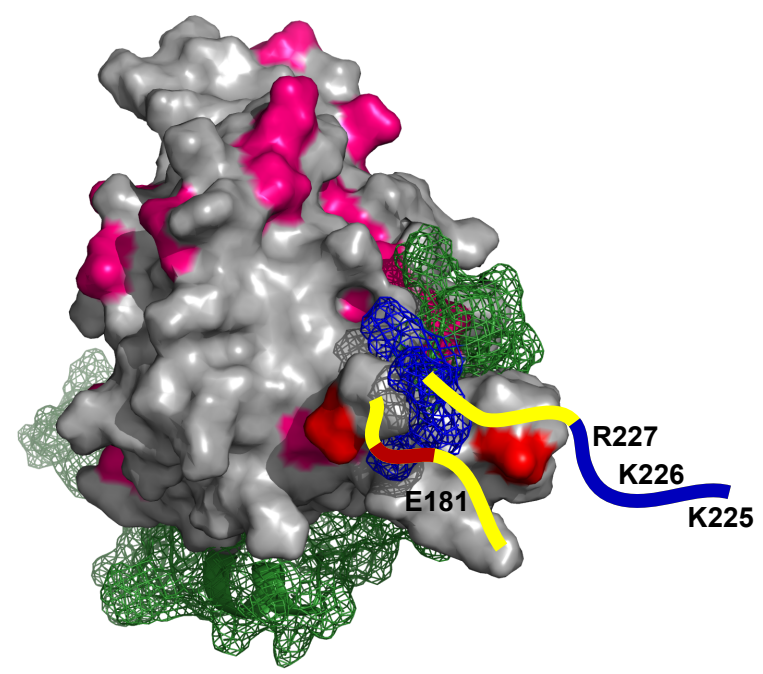

B

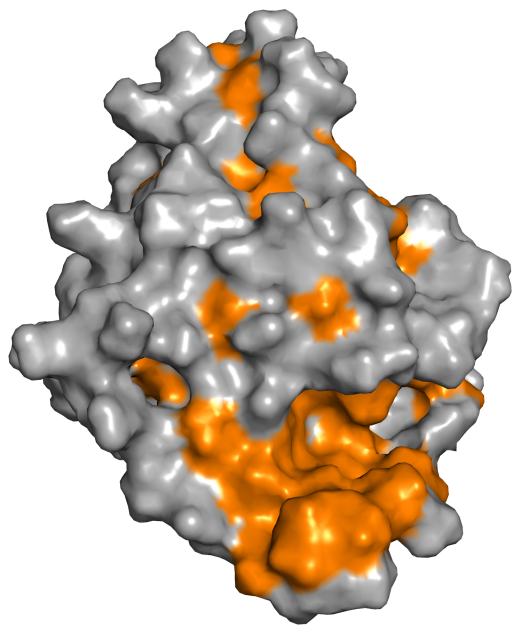

D

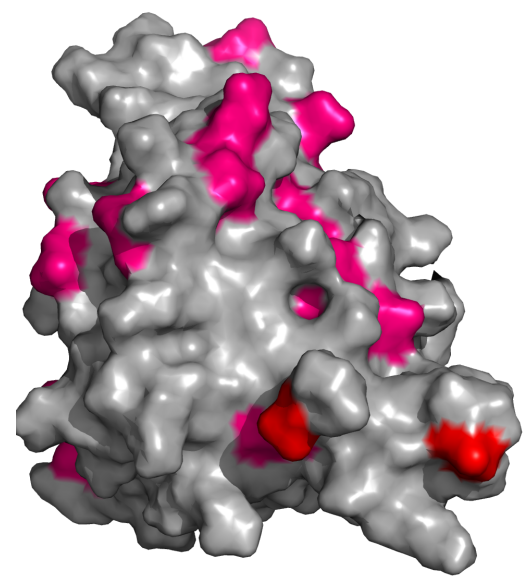


Figure 6

A

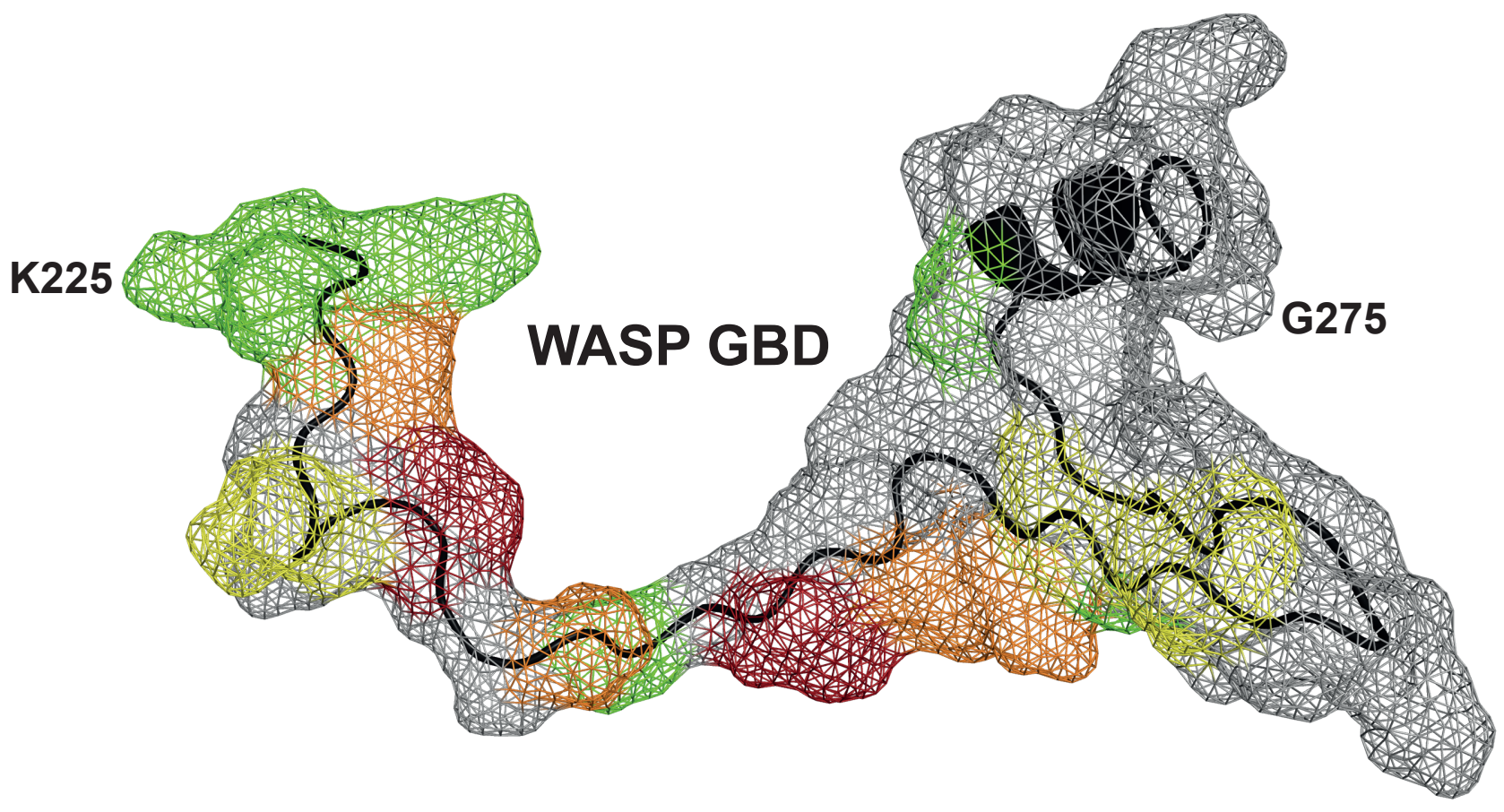

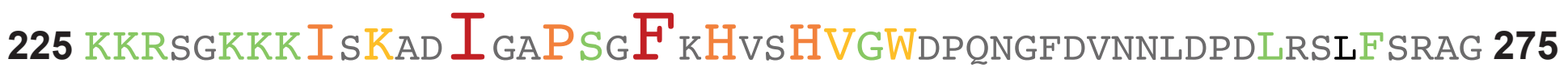

B

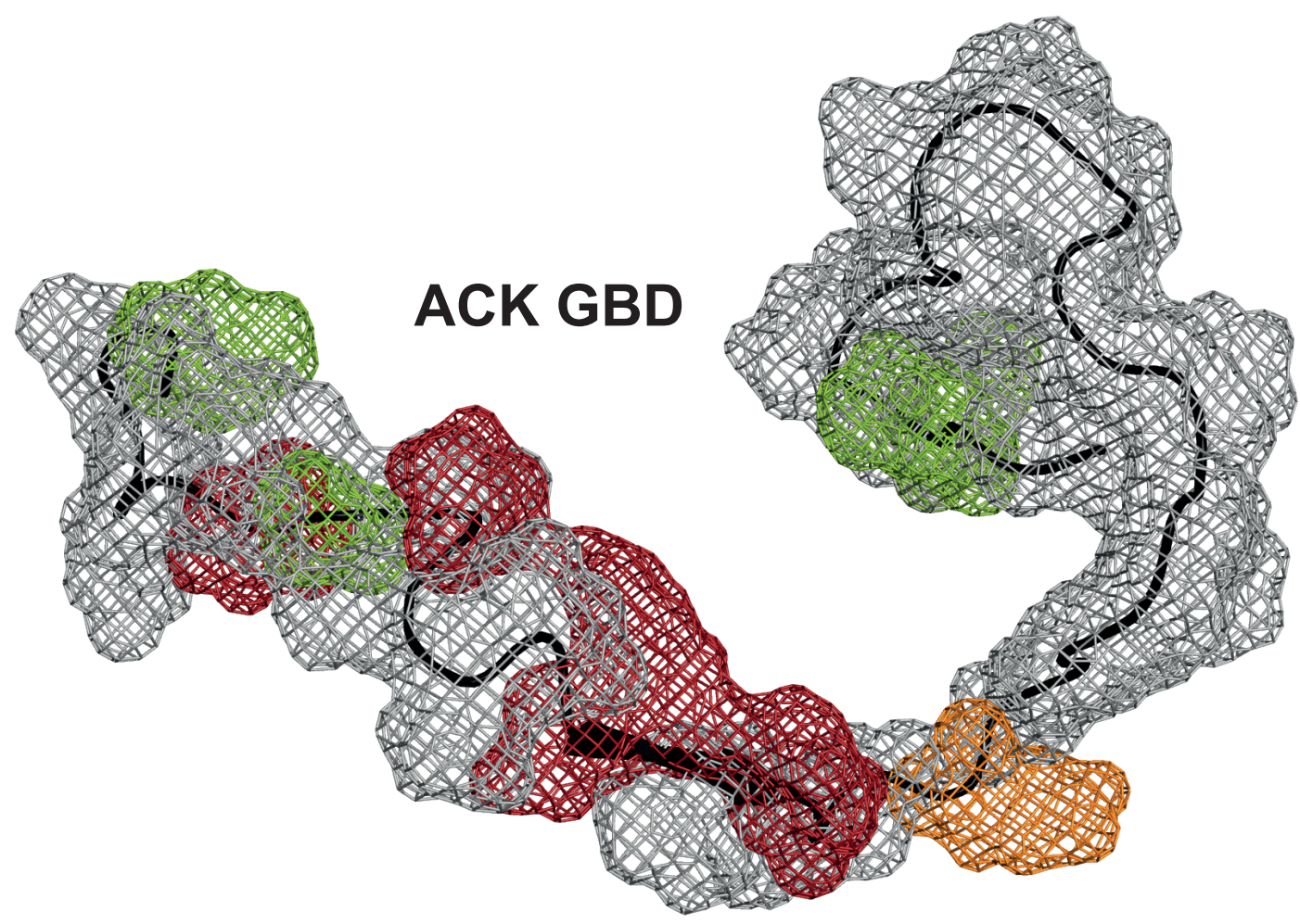

448 GL SAQD $I_{S Q} P_{L Q N} S_{I} H_{T G H} H_{\text {GDSDPRHCWGFPRIDELYLGN }} 489$ 